\title{
Research
}

\section{Predictive values of referrals for transient ischaemic attack from first-contact health care:}

\author{
a systematic review
}

\begin{abstract}
Background

Over 150000 cases of suspected transient

ischaemic attack (TIA) are referred to

outpatient clinics in England each year. The majority of referrals are made by GPS.
\end{abstract}

\section{Aim}

This study aimed to identify how many patients referred to a TIA clinic actually have TIA (that is, calculate the positive predictive value [PPV] of first-contact healthcare referral) and to record the alternative diagnoses in patients without TIA, in order to determine the optimal service model for patients with suspected TIA.

\section{Design and setting}

A systematic review of TIA clinic referrals from first-contact health professionals (GPS and emergency department [ED] doctors) was undertaken.

\section{Method}

Four databases were searched using terms for TIA and diagnostic accuracy. Data on the number of patients referred to a TIA clinic who actually had a TIA (PPVs) were extracted. Frequencies of differential diagnoses were recorded, where reported. Study quality was assessed using the QUADAS-2 tool.

\section{Results}

Nineteen studies were included and reported sufficient information on referrals from GPs and ED doctors to derive PPVs I $n=15935$ referrals). PPVs for TIA ranged from 12.9\% to $72.5 \%$. A formal meta-analysis was not conducted due to heterogeneity across studies. Of those not diagnosed with TIA, approximately half of the final diagnoses were of neurological or cardiovascular conditions.

\section{Conclusion}

This study highlights the variation in prevalence of true vascular events in patients referred to TIA clinics. For patients without a cerebrovascular diagnosis, the high prevalence of conditions that also require specialist investigations and management are an additional burden on a care pathway that is primarily designed to prevent recurrent stroke. Service commissioners need to assess whether the existing outpatient provision is optimal for people with pathologies other than cerebrovascular disease.

\section{Keywords}

diagnosis; general practitioners; ischaemic attack, transient; predictive value of tests primary health care; stroke.

\section{INTRODUCTION}

A transient ischaemic attack (TIA) is a temporary focal neurological disturbance due to an interruption in the blood supply to an area of the brain. ${ }^{1}$ The term 'transient neurological symptoms' is used to describe the broad range of symptoms that may occur following a TIA or another condition that may mimic TIA. There is no goldstandard clinical test that can be used to diagnose a TIA or stroke based on symptomology; diagnosis of TIA is based on the assessment of symptoms and adequate investigation by a clinician. Historically, TIA symptoms would need to resolve within 24 hours to be classified as TIA and not a minor stroke; however, in 2009 a tissuebased definition of TIA was proposed:

'Transient ischemic attack (TIA): a transient episode of neurological dysfunction caused by focal brain, spinal cord, or retinal ischemia, without acute infarction.'

In practice, the time-based definition may be the more-operable, working definition, because identification of infarcts requires imaging and not all TIA clinic attendees are imaged.

The incidence of transient neurological symptoms is high - estimated at 190 cases per 100000 population ${ }^{2}$ - and clinic referral rates amount to approximately 16 per

R Kandiyali, MA, MSc, PhD, senior research associate, Bristol Medical School, University of Bristol, Bristol. DS Lasserson, MA, MD, FRCP Edin, MRCGP, professor of ambulatory care, Institute of Applied Health Research, College of Medical and Dental Sciences, University of Birmingham, Birmingham. P Whiting, PhD, senior research fellow, Bristol Medical School, University of Bristol, Bristol, and NIHR CLAHRC West, Bristol. A Richards, MA, MCILIP, information scientist, NIHR CLAHRC West, Bristol. J Mant, MD, FFPH, professor of primary care research, Primary Care Unit, Department of Public Health and Primary Care, University of Cambridge, Cambridge.
10000 patients every year. ${ }^{3}$ Outpatient TIA clinics are well equipped to identify and treat TIA and minor stroke, but only a proportion of suspected TIA cases will be confirmed. ${ }^{4}$ Those patients not presenting with a TIA may, nevertheless, experience adverse health consequences. ${ }^{5}$

The clinical assessment for TIA can be complex because the symptoms are transient and there are no persisting signs on examination to guide the referring clinician. Previously, a brief review of predictive values in TIA was undertaken, ${ }^{6}$ but an update is timely as more data have been published to inform estimates of accuracy. Richer data now also exists on alternative diagnoses in this complex clinic population. The objective of this study was to evaluate the positive predictive values (PPVs) associated with first-contact healthcare referral - that is, those made by GPs or emergency department (ED) doctors - to a TIA clinic, and to describe the alternative diagnoses in referred patients.

\section{METHOD}

\section{Data sources}

Four databases (MEDLINE, EMBASE, the Cochrane Database of Systematic Reviews, and the Database of Abstracts of Reviews of Effects) were searched from 1989 to week 28 of 2016, using terms for TIA combined with a diagnostic filter lavailable from the

\section{Address for correspondence}

Bristol Medical School, University of Bristol, Oakfield House, Oakfield Place, Bristol, BS8 2BN, UK.

E-mail: rebecca.kandiyaliabristol.ac.uk

Submitted: 29 March 2017; Editor's response: 8 May 2017; final acceptance: 19 June 2017. (c)British Journal of General Practice

This is the full-length article (published online 21 Nov 2017) of an abridged version published in print. Cite this version as: Br J Gen Pract 2017; DOI: https://doi.org/10.3399/10.3399/bjgp17X693677 


\section{How this fits in}

The positive predictive value of a referral to a transient ischaemic attack (TIA) clinic has previously been described in selected populations in single studies. The systematic review presented here found that $12.9-72.5 \%$ of clinic referrals had a confirmed TIA; this was usually $\geq 50 \%$ when a composite (TIA or minor stroke) reference standard was used. Alternative diagnoses suggest that the total population with transient neurological symptoms may represent a susceptible population for further investigations and treatment that is not presently discussed in UK stroke and TIA guidance. Commissioners should ensure that TIA services can meet the needs of a heterogeneous patient group.

authors on request). The Bachmann filter (adapted to run on each database) was used - this has been identified as one of the most sensitive diagnostic filters available, with acceptable precision. ${ }^{7}$ Additional papers were sought by screening the citations of retrieved studies. All data screening, extraction, and full-text assessment were done by a single reviewer and checked in detail by a second.

\section{Inclusion criteria}

Primary studies of any design, conference abstracts, and systematic reviews reporting information necessary to derive PPVs of TIA diagnosis from first-contact health professionals (primarily GPs or ED doctors) were included. If PPVs were reported in more than one study, duplicate values were not reported. When there was a duplication of reporting, preference was given to fulltext studies that report the most detail with respect to the application of the index test and reference standard.

\section{Data extraction and study quality assessment}

Data were extracted on the:

- type of study;

- geographical location;

- method of patient selection;

- age of population; and

- number of patients included in the study. Information was also collected on:

- positive and negative diagnoses;

- frequencies of unverified diagnoses;

- which reference standard the study applied (TIA alone or TIA and minor stroke); and

- what definition of TIA was used (tissue- or time-based definition).

Systematic reviews were identified as a source of relevant studies. QUADAS-2 was used to assess the risk of bias and applicability of included studies. ${ }^{8}$ The frequencies of differential diagnoses for false-positive TIAs were also recorded, and the details of all nonTIA/stroke diagnoses tabulated.

\section{Statistical analysis and synthesis}

For each study, the PPV was calculated as the number of true positives divided by the sum of true and false positives (that is, the total number of patients referred to the clinic). The binomial exact standard errors were calculated if the standard error of the PPV was not reported. Due to high unexplained variation in the underlying prevalence of TIA, it was decided that a summary PPV would not be estimated. Forest plots were used to display the individual study estimates of PPV, with 95\% confidence intervals (Cls) analysing the target conditions (TIA and the composite outcome of TIA and minor stroke) separately. Although the main analysis reports results for full texts only, a sensitivity analysis including conference abstracts was carried out to examine the robustness of the results.

\section{RESULTS}

The search identified 3924 unique records. Of these, and a further three records identified by screening the references, 19 full texts met the eligibility criteria (Figure 1). Eleven conference abstracts also met the inclusion criteria and were included in the sensitivity analysis. Study characteristics are presented in Table 1.

Ten studies were conducted in the UK, three in Ireland, two in Australia, two in Portugal, one in Spain, and one in France. All patients identified were TIA clinic referrals/attendees, using consecutive or all referrals within a given timeframe. In total, 19 studies provided sufficient information to calculate the PPV for at least one of the reference diagnoses (TIA and/or the composite reference diagnosis of TIA and minor stroke). The number of suspected cases of TIA referred from, or including, GPs (18 of 19 studies) ranged from 52 to 3533 (Table 1). 9.10

\section{Specialist diagnosis (reference standard)}

In all cases, the reference standard was the clinical diagnosis of the stroke physician in clinic. Several studies reported that the 


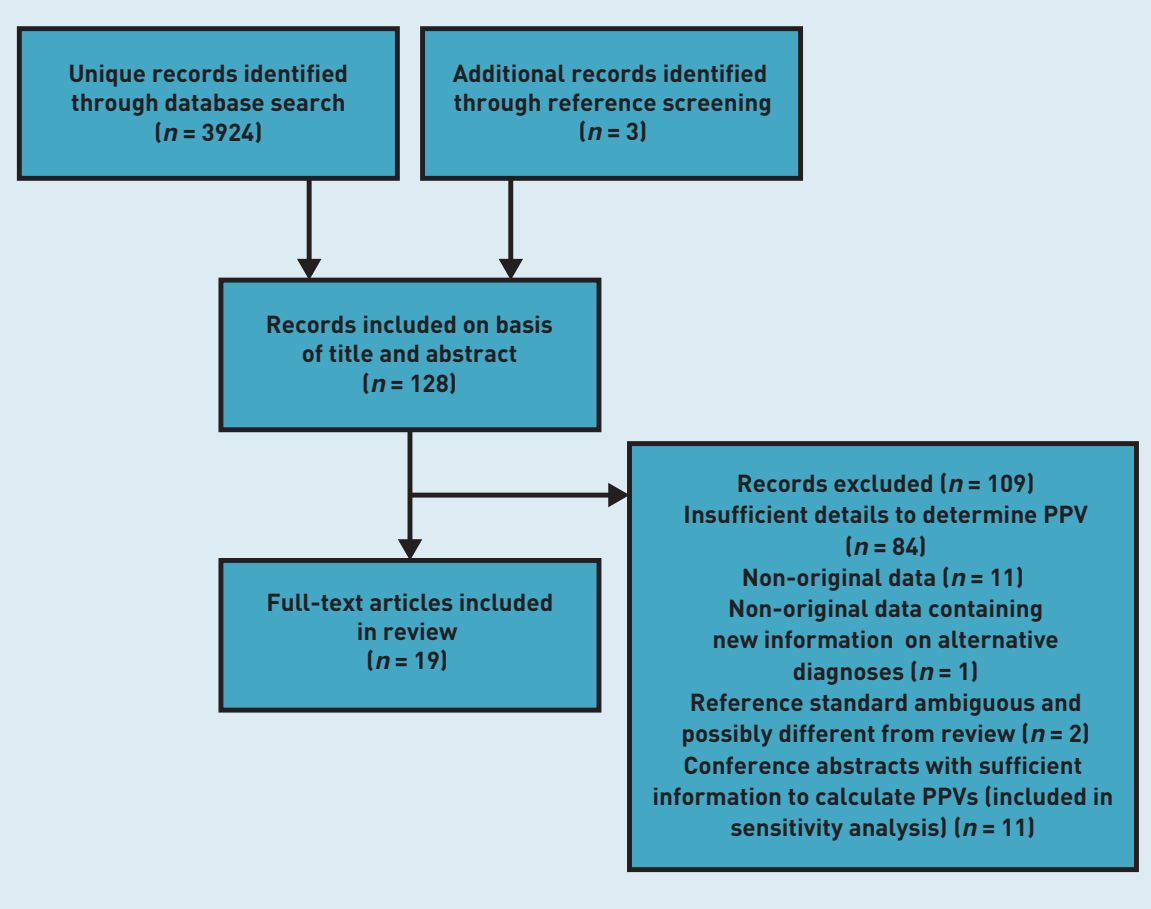

Figure 1. Study selection process. $P P V=$ positive predictive value.

Figure 2. PPVs of first-contact healthcare diagnosis in $T I A$ and stroke. $\mathrm{Cl}=$ confidence interval. $E D=$ emergency department. $P P V=$ positive predictive value. $T I A=$ transient ischaemic attack assessment of TIA was standardised at their clinic and/or of additional retrospective notes review to confirm the diagnosis made by a senior stroke/vascular specialist. Studies dichotomised diagnoses into two outcomes:
- TIA, which sometimes included minor stroke; and
- not TIA.

Almost all studies used the time-based definition of TIA, even when the later tissuebased definition was available, but there was one exception: one conference abstract included in the sensitivity analysis ${ }^{24}$ used the tissue-based definition of TIA.

\section{Differential diagnoses}

Twelve of the included studies reported on the final diagnoses received by patients, although one study did not report sufficient information to determine frequencies for all alternative diagnoses. ${ }^{25}$ The frequency of alternative diagnoses, where reported, are shown in Table 2 .

The range of conditions diagnosed includes those for which guidance from the National Institute for Health and Care Excellence recommends assessment by an appropriately trained specialist, such as for multiple sclerosis, epilepsy, and cardiac arrhythmias. The most common diagnoses were seizure, syncope, transient global amnesia, and migraine/tension headache (Table 2).

\section{Unexplained diagnoses}

The majority -14 out of 19 - of studies did not provide clear information on the number of patients for whom there was no clear diagnosis following referral to a TIA clinic (Table 2). Several studies had a 'possible TIA' category ${ }^{18,26}$ featuring symptoms that were broadly consistent with, but not clearly diagnostic for, TIA, as well as a 'non-TIA' category when this was not the case. As the diagnosis was essentially unconfirmed in these cases, the analysis in this review treated possible TIA as essentially unexplained, that is, negative cases in the analysis of PPV.

\section{PPVs of TIA from first-contact health care}

The proportion of referred patients with a final diagnosis of TIA and/or minor stroke ranged from $22.0 \%$ to $77.9 \%$ (Figure 2), and from $12.9 \%$ to $72.5 \%$ of patients with a final diagnosis of TIA (Figure 3). The distribution of PPV estimates appeared to differ depending on the reference standard -13 out of 18 studies had a PPV of $\geq 50 \%$ for a combined TIA and minor stroke outcome, but only four of 18 studies had a PPV of $\geq 50 \%$ when the reference standard was just TIA.

\section{Assessment of study quality}

Application of the QUADAS-2 checklist yielded similar results across studies, with all having a high risk of bias in the referencestandard domain. The bias relates to the absence of a 'gold-standard' test and the 


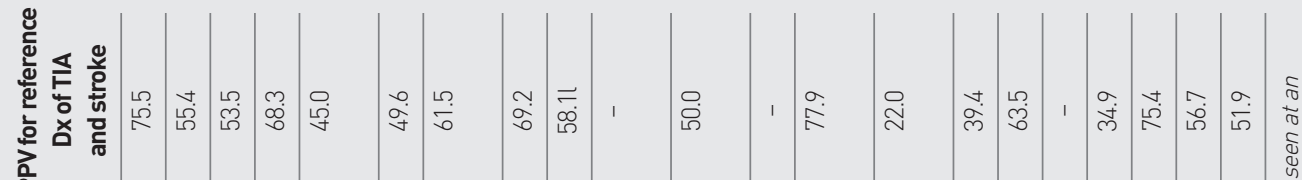
a

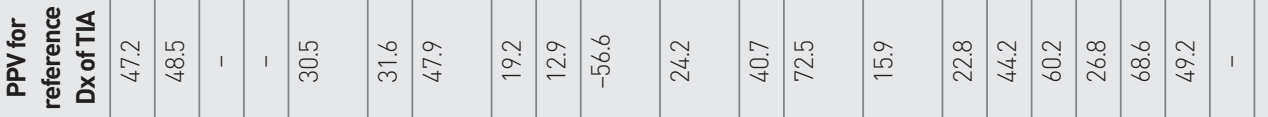

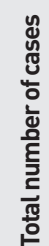

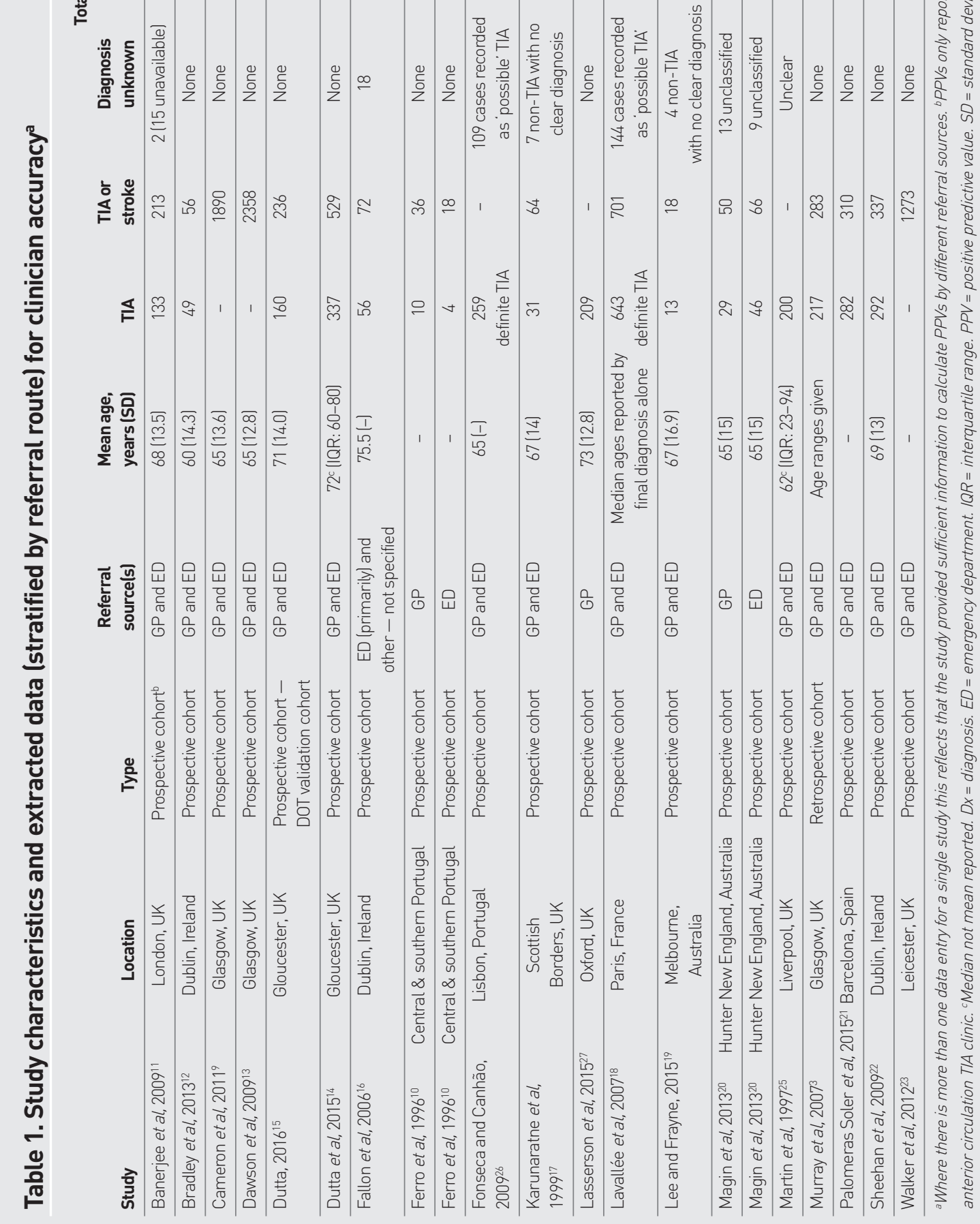

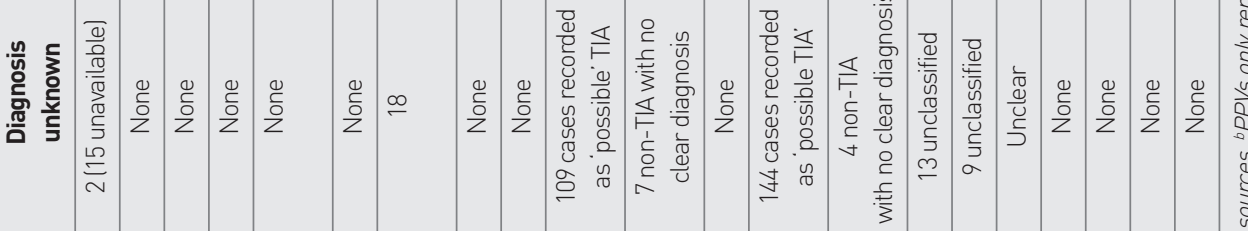

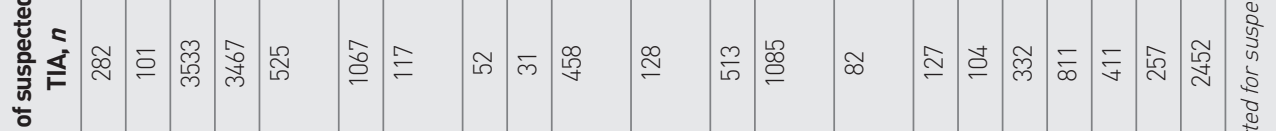
贾 


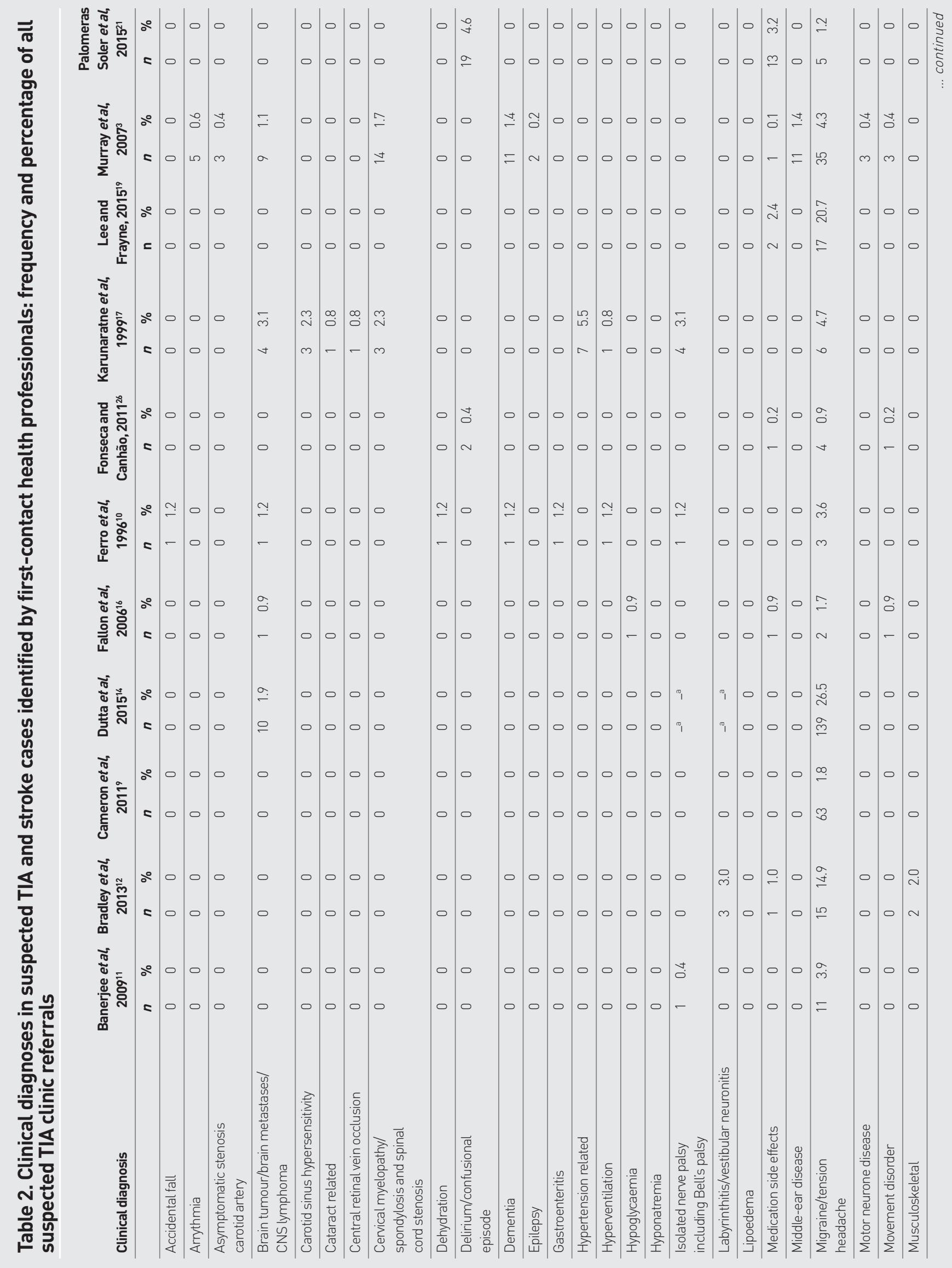




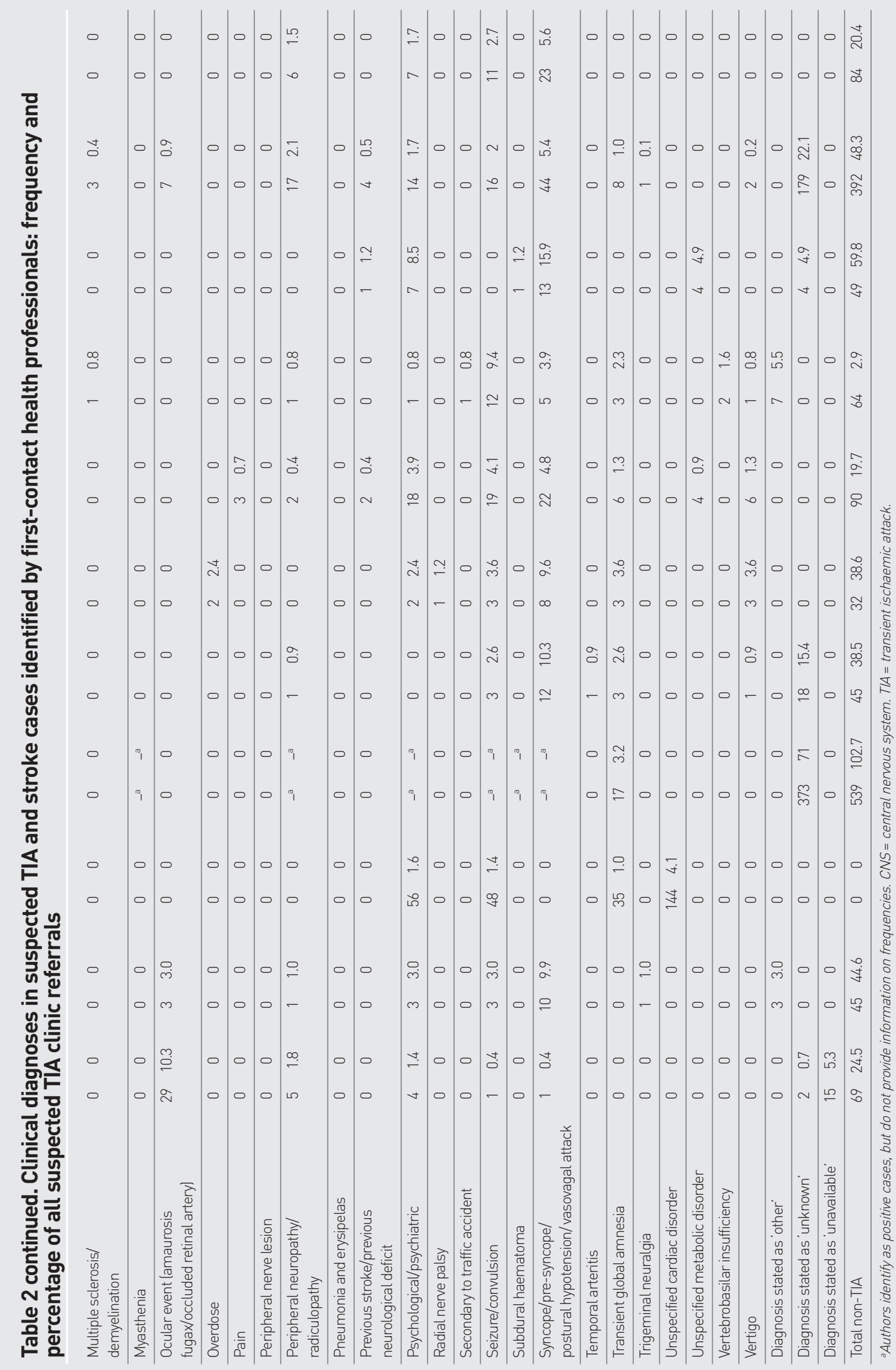


Figure 3. PPVs of first-contact healthcare diagnosis in $\mathrm{TIA} . \mathrm{Cl}=$ confidence interval. $\mathrm{ED}=$ emergency department. $P P V=$ positive predictive value. $T I A=$ transient ischaemic attack
Figure 4. PPVs of first-contact healthcare diagnosis in TIA and stroke (sensitivity analysis including original data contained in conference abstracts). Letters ${ }^{a}$ Conference abstracts not included in main analysis. $\mathrm{Cl}=$ confidence interval. $E D=$ emergency department. $P P V=$ positive predictive value. $T I A=$ transient ischaemic attack.

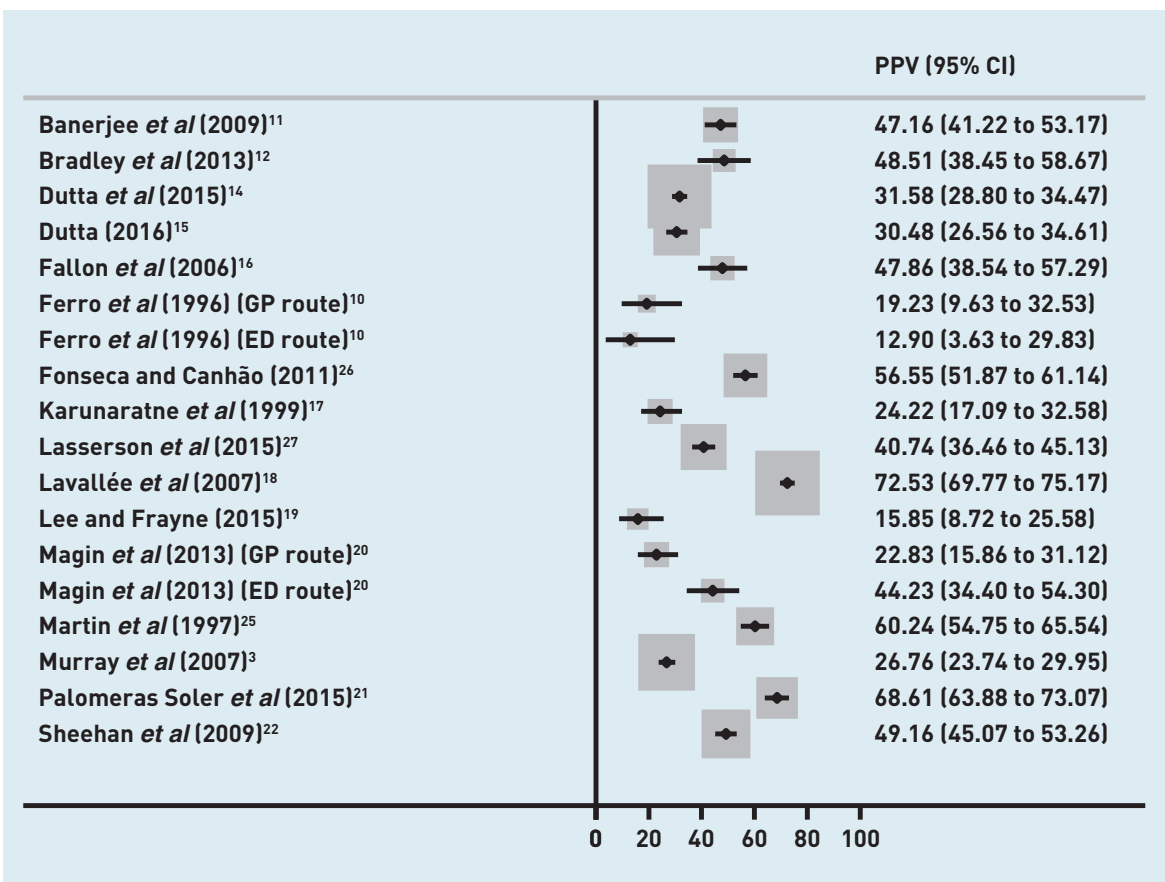

diagnostician knowing that the patients were referred as having suspected TIA.

\section{Influence of referral source and referral} criterion

The majority of studies -15 out of 19 included all referrals and did not report on the composition of referrals (GP or ED doctorl and/or provide sufficient data to calculate PPVs by referral source. It is plausible that studies may have included referrals from other sources such as ophthalmology and secondary care, but reporting on this issue was scant.

Two studies ${ }^{10,20}$ provided sufficient information to calculate PPVs according to two referral routes (GP or ED doctor) and a further study provided information on PPVs for referrals purely from GPs. ${ }^{27}$ One study gave PPVs predominantly from

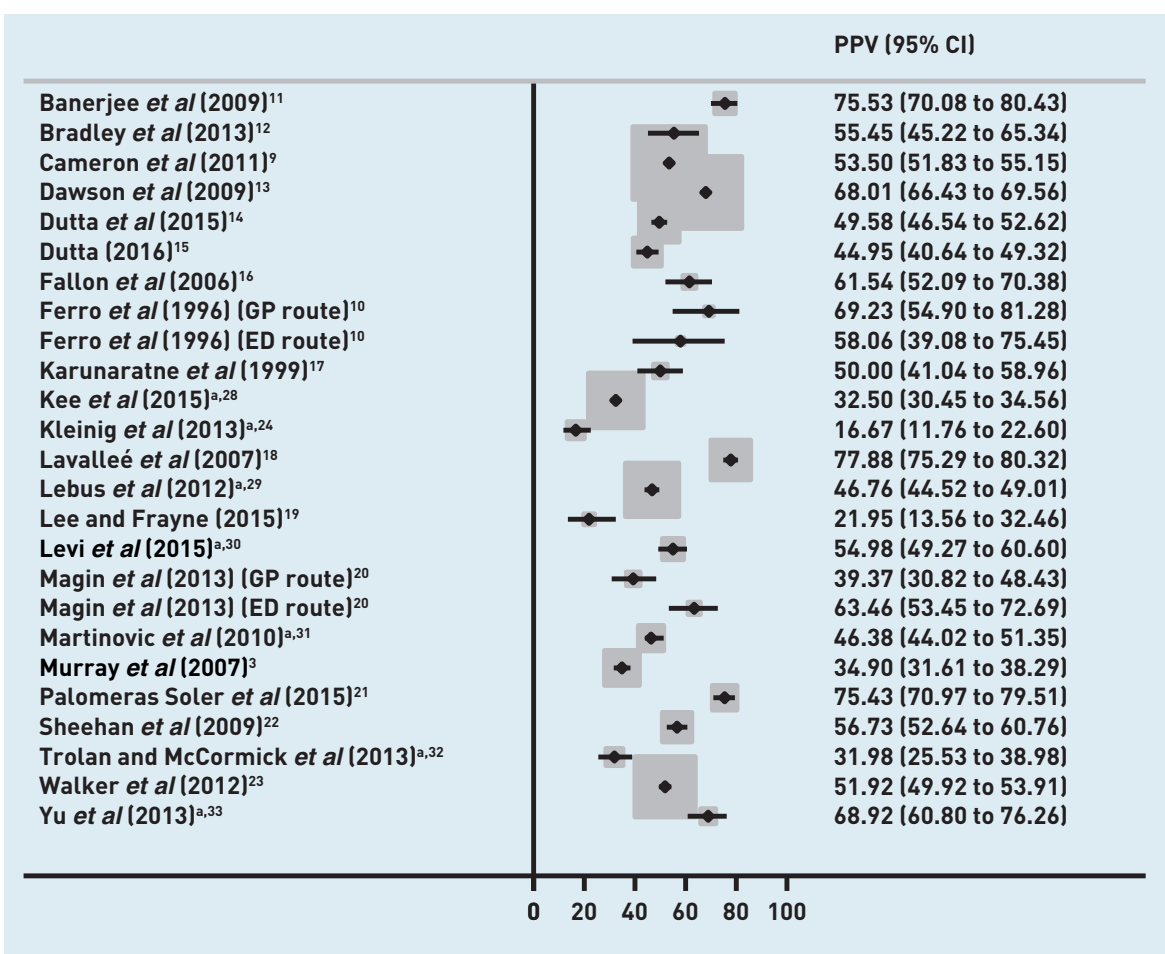


Figure 5. PPVs of first-contact healthcare diagnosis in TIA (sensitivity analysis including original data contained in conference abstracts). a Conference abstracts not included in main analysis. $\mathrm{Cl}=$ confidence interval. $E D=$ emergency department. $P P V=$ positive predictive value. $T I A=$ transient ischaemic attack.

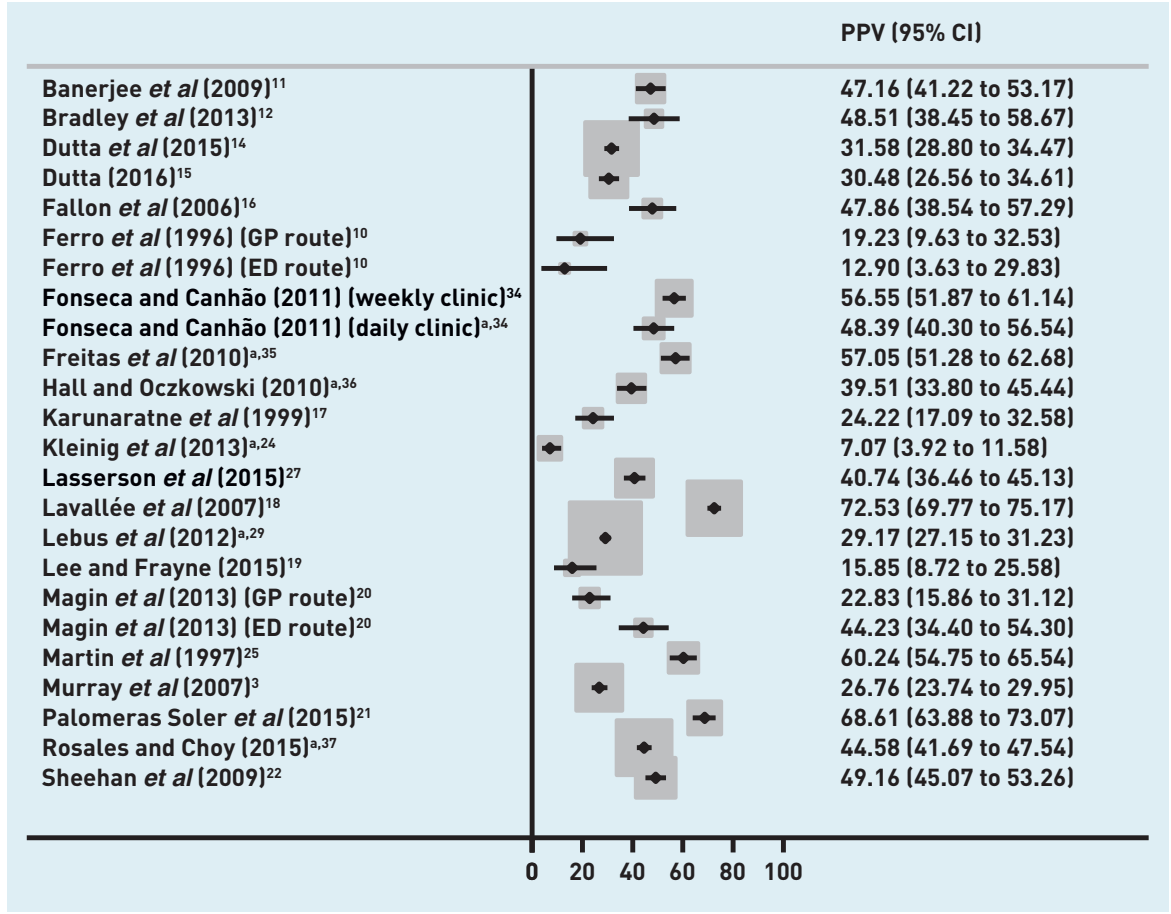

an ED setting, ${ }^{16}$ whereas all other studies appeared to have largely comprised referrals from GPs. With the exception of one small study, ${ }^{10}$ the PPVs appear lower in GP referrals than in referrals from ED doctors (Table 1; Figures 2 and 3). Only one study, which restricted itself to suspected anterior circulation TIA events, ${ }^{11}$ described specific referral criteria.

\section{Impact of including conference abstracts}

In general, interpretation of the results did not change when conference abstracts were included (Figures 4 and 5), 24,28-37 although Kleinig et $a^{24}$ had much lower PPVs compared with the other studies for both the combined outcome $116.7 \%$, $95 \% \mathrm{Cl}=11.8$ to 22.6 , Figure 4$)$ and $\mathrm{TIA}$ alone $(7.1 \%, 95 \% \mathrm{Cl}=3.9$ to 11.6 , Figure 5$)$. This study was set in a magnetic resonance imaging-based referral clinic using the tissue-based reference standard.

\section{DISCUSSION}

\section{Summary}

This review has identified considerable variability in PPVs for TIA across studies. The subset of studies identified, which report on alternative diagnoses, highlights the predominance of additional neurological and cardiological diseases that are TIA mimics and require specialist assessment, either within the TIA service or at a subsequent specialty clinic attendance.
Although the review demonstrates a variation in PPVs across studies, it could be that this is explained by a combination of referral source and diagnostic criteria, study age, and/or the cardiovascular event being diagnosed - for instance, some evidence was found to suggest that PPVs in primary care populations may be lower than in those whose referral was made by an ED doctor. However, inference about the possible influence of referral source and referral criterion is difficult because of other study differences.

PPVs also tended to be higher in studies conducted in recent years, which might reflect a change in operation of the diagnostic criteria and improving recognition of symptoms by doctors over time.

Studies that included stroke had higher PPVs; this might reflect the broader diagnostic criteria or that GPs and ED doctors may be more likely to correctly identify a stroke due to the persistent nature of the deficit.

\section{Strengths and limitations}

As there was no assessment of patients who were not thought by first-contact health professionals to have experienced a TIA, it was not possible for the authors to ascertain how many people with TIAs were missed. As such, it was not possible to compute sensitivity or specificity. This means that it was also not possible to interpret whether high predictive values were associated with 


\section{Funding}

This study was unfunded; however, Rebecca Kandiyali previously carried out a literature review on this topic as part of a University of Birmingham medical school studentship and Daniel Lasserson is supported by the NIHR Oxford Diagnostic Evidence Cooperative. Penny Whiting and Alison Richards were supported by the National Institute for Health Research (NIHR) Collaboration for Leadership in Applied Health Research and Care (CLAHRC) West at University Hospitals Bristol NHS Foundation Trust. The views are those of the authors and not necessarily those of the NIHR, NHS, or Department of Health. The funders had no role in the design of the study, data collection and analysis, decision to publish, or preparation of the manuscript.

\section{Ethical approval}

Not applicable.

\section{Provenance}

Freely submitted; externally peer reviewed.

\section{Competing interests}

The authors have declared no competing interests.

\section{Acknowledgements}

The authors would like to thank Professor Willie Hamilton for commenting on the study design.

\section{Discuss this article}

Contribute and read comments about this article: bjgp.org/letters

higher referral thresholds (which are likely to be associated with lower sensitivity - that is, more TIAs missed by first-contact health professionals). It also means the prevalence of TIA in the population seen by first-contact health professionals cannot be computed, which is a key determinant of predictive value.

Although the authors believed the reference standard to be acceptable - in all cases it was analogous to how diagnoses are made in practice - specialists were not blind to the index GP/ED doctor diagnosis; this might have led to more non-TIAs being misclassified as TIAs.

Although not limitations as such, there are some caveats to inference on the basis of PPVs. The PPVs reported are at study level. Each study reflects the practice of multiple clinicians, which may vary considerably. In addition, a PPV does not indicate whether the referral to the TIA clinic was appropriate for the patient, and/or whether a more appropriate action should have been taken.

The decision to explore potential publication bias via a sensitivity analysis of conference abstracts is a strength. Predictive values for TIA were similar, suggesting that publication bias is unlikely to be a major issue.

\section{Comparison with existing literature}

This is the first review of predictive values from first-contact health care and as such it summarises the literature. However, the PPV of a stroke referral from a review of the literature is about $74 \%,{ }^{4}$ which is higher than any individual PPV for TIA. This may be due to easier recognition of stroke with both persisting neurological deficit and a more severe clinical phenotype. It is interesting to note that the PPVs in this study are higher than those for cancer, where there is an accelerated referral process in order to expedite diagnosis and treatment. ${ }^{38-40}$ However, the complexity of diagnosis and ongoing management of the other conditions that are found among TIA referrals mandates that other specialties are involved in service delivery, an issue that has less of an impact on organ-specific cancer referral pathways.

\section{Implications for practice}

PPVs are a key statistic used in predictive risk modelling and the planning of prevention services. ${ }^{41}$ The use of PPVs as statistics for planning TIA services has been contested ${ }^{42}$ because patients with transient symptoms that are not a result of TIA have been recognised as a similarly morbid population to those with true TIA. ${ }^{43-45}$ Clinical need is, therefore, not limited to confirmed TIAs but to the broader populace with transient symptoms. The dual findings of this review - relatively high but variable predictive values and a predominance of cardiovascular pathologies - suggest that active risk-factor management, including early initiation of antiplatelet agents, is still appropriate to mitigate early recurrent stroke risk after initial suspicion of TIA. ${ }^{46}$ This study shows that TIA specialist services need to handle a broad range of diagnoses, not only TIA. Many of the most common alternative diagnoses could benefit from appropriate specialty input - the challenge for service commissioners is how best to deliver comprehensive care for patients who present with transient neurological symptoms. Although the TIA clinic is well placed to manage the hyper-acute risk of recurrent stroke, it may not be the optimal configuration in terms of specialist assessment for the range of neurological, cardiological, and psychiatric conditions that also require ongoing care. 


\section{REFERENCES}

1. Easton JD, Saver JL, Albers GW, et al. Definition and evaluation of transient ischemic attack: a scientific statement for healthcare professionals from the American Heart Association/American Stroke Association Stroke Council; Council on Cardiovascular Surgery and Anesthesia; Council on Cardiovascular Radiology and Intervention; Council on Cardiovascular Nursing; and the Interdisciplinary Council on Peripheral Vascular Disease. The American Academy of Neurology affirms the value of this statement as an educational tool for neurologists. Stroke 2009; 40(6): 2276-2293.

2. Gibbs RG, Newson R, Lawrenson R, et al. Diagnosis and initial management of stroke and transient ischemic attack across UK health regions from 1992 to 1996: experience of a national primary care database. Stroke 2001; $\mathbf{3 2 ( 5 )}$ : 1085-1090.

3. Murray S, Bashir K, Lees KR, et al. Epidemiological aspects of referral to TIA clinics in Glasgow. Scott Med J 2007; 52(1): 4-8

4. Gibson LM, Whiteley W. The differential diagnosis of suspected stroke: a systematic review. J R Coll Physicians Edinb 2013; 43(2): 114-118.

5. Bos MJ, van Rijn MJ, Witteman JC, et al. Incidence and prognosis of transient neurological attacks. JAMA 2007; 298(24): 2877-2885.

6. Mant J, Ryan R, McManus R, et al. What is the optimum model of service delivery for transient ischaemic attack? Report for the National Co-ordinating Centre for NHS Service Delivery and Organization R\&D (NCCSDO). 2008. http://www.netscc.ac.uk/hsdr/files/project/SDO_FR_08-1504-112_V01.pdf laccessed 19 Oct 2017).

7. Bachmann LM, Coray R, Estermann P, Ter Riet G. Identifying diagnostic studies in MEDLINE: reducing the number needed to read. J Am Med Inform Assoc 2002; 9(6): 653-658.

8. Whiting PF, Rutjes AW, Westwood ME, et al. QUADAS-2: a revised tool for the quality assessment of diagnostic accuracy studies. Ann Intern Med 2011; 155(8): 529-536

9. Cameron AC, Dawson J, Quinn TJ, et al. Long-term outcome following attendance at a transient ischemic attack clinic. Int J Stroke 2011; 6(4): 306-311.

10. Ferro JM, Falcão I, Rodrigues G, et al. Diagnosis of transient ischemic attack by the nonneurologist: a validation study. Stroke 1996; 27(12): 2225-2229.

11. Banerjee S, Natarajan I, Biram R, et al. FAST-TIA: a prospective evaluation of a nurse-led anterior circulation TIA clinic. Postgrad Med J 2009; 85(1010): 637-642.

12. Bradley D, Cronin S, Kinsella JA, et al. Frequent inaccuracies in ABCD2 scoring in non-stroke specialists' referrals to a daily Rapid Access Stroke Prevention service. J Neurol Sci2013; 332(1-2): 30-34

13. Dawson J, Lamb KE, Quinn TJ, et al. A recognition tool for transient ischaemic attack. Q J Med 2009; 102(1): 43-49

14. Dutta D, Bowen E, Foy C. Four-year follow-up of transient ischemic attacks, strokes, and mimics: a retrospective transient ischemic attack clinic cohort study. Stroke 2015; 46(5): 1227-1232.

15. Dutta D. Diagnosis of TIA (DOT) score - design and validation of a new clinical diagnostic tool for transient ischaemic attack. BMC Neurol 2016; 16(1): 20.

16. Fallon C, Noone I, Ryan J, et al. Assessment and management of transient ischaemic attack - the role of the TIA clinic. Ir J Med Sci 2006; 175(3): 24-27.

17. Karunaratne PM, Norris CA, Syme PD. Analysis of six months' referrals to a 'one-stop' neurovascular clinic in a district general hospital: implications for purchasers of a stroke service. Health Bull 1999; 57(1): 17-28.

18. Lavallée PC, Meseguer $\mathrm{E}$, Abboud $\mathrm{H}$, et al. A transient ischaemic attack clinic with round-the-clock access (SOS-TIA): feasibility and effects. Lancet Neurol 2007; 6(11): 953-960.

19. Lee W, Frayne J. Transient ischaemic attack clinic: an evaluation of diagnoses and clinical decision making. J Clin Neurosci2015; 22(4): 645-648.

20. Magin P, Lasserson D, Parsons M, et al. Referral and triage of patients with transient ischemic attacks to an acute access clinic: risk stratification in an Australian setting. Int J Stroke 2013; 8(Suppl A100): 81-89.

21. Palomeras Soler E, Fossas Felip P, Cano Orgaz AT, et al. Rapid assessment of transient ischaemic attack in a hospital with no on-call neurologist. [Spanish]. Neurologia 2015; 30(6): 325-350.

22. Sheehan OC, Merwick A, Kelly LA, et al. Diagnostic usefulness of the ABCD2 score to distinguish transient ischemic attack and minor ischemic stroke from noncerebrovascular events: the North Dublin TIA Study. Stroke 2009; 40(11): 3449-3454
23. Walker J, Isherwood J, Eveson D, Naylor AR. Triaging TIA/minor stroke patients using the ABCD2 score does not predict those with significant carotid disease. Eur J Vasc Endovasc Surg 2012; 43(5): 495-498

24. Kleinig $T$, Hall $L$, Jannes J, Dowie G. There's (almost) no such thing as a TIA; high rates of TIA-mimics and minor stroke in a tertiary MRI- and emergency referral-based TIA service. Int J Stroke 2013; 8(Suppl 1): 48.

25. Martin PJ, Young G, Enevoldson TP, Humphrey PRD. Overdiagnosis of TIA and minor stroke: experience at a regional neurovascular clinic. Q J Med 1997; 90(12): 759-763

26. Fonseca AC, Canhão P. Diagnostic difficulties in the classification of transient neurological attacks. Eur J Neurol 2011; 18(4): 644-648.

27. Lasserson DS, Mant D, Hobbs FD, Rothwell PM. Validation of a TIA recognition tool in primary and secondary care: implications for generalizability. Int J Stroke 2015; 10(5): 692-696.

28. Kee Y, Negansan C, Mahmood S, Lawrence E. Fast +ve vs Fast -ve: an analysis of patients presenting to TIA clinic. Int J Stroke 2015; 10(Suppl 5): 55.

29. Lebus C, Prabhakaran M, Mitchell J, et al. The ABCD2 score as a predictor of prognosis but also accurate diagnosis of TIA in a large teaching hospital service. Cerebrovasc Dis 2012; 33(Suppl 2): 524-525.

30. Levi C, Davey A, Lasserson D, et al. Presentation patterns of patients with transient ischemic attack (TIA) and minor stroke, compared with those of stroke/TIA mimics. Int J Stroke 2015; 10(Suppl 2): 362.

31. Martinovic $\mathrm{O}$, Baht $\mathrm{H}$, Balogun I, et al. An appointment based TIA service is unable to accommodate all high risk patients <24hrs. Cerebrovasc Dis 2010 29(Suppl 2): 238.

32. Trolan C, McCormick M. Prioritisation of referrals to a district general neurovascular clinic: role of ABCD2. Ir J Med Sci 2013; 182(Suppl 6): S281.

33. Yu CT, Tam YM, Tang SK, et al. A prospective evaluation of diagnostic yield of transient ischemic attack (TIA) in a nurse-led TIA clinic in Hong Kong. Cerebrovasc Dis 2013; 36(Suppl 1): 29

34. Fonseca M, Canhão P. Early vascular risk after TIA: comparison between a weekly and daily TIA clinic. J Neurol 2011; 258(1 Suppl): S34.

35. Freitas J, Damasio J, Magalhaes R, et al. Strokes ABCD2 score in the distinction between vascular and non-vascular transient neurologic attack. Cerebrovasc Dis 2010; 29(Suppl 2): 238-239.

36. Hall C, Oczkowski W. Utility of the ABCD and ABCD2 scores in identifying true TIA events in the emergency department. CJEM 2010; 12(3): 232.

37. Rosales CF, Choy LGY. Three year TIA clinic audit from a UK district general hospital. Cerebrovasc Dis 2015; 39(Suppl 2): 173

38. Hjertholm P, Moth G, Ingeman ML, Vedsted P. Predictive values of GP' suspicion of serious disease: a population-based follow-up study. $\mathrm{Br} J \mathrm{Gen}$ Pract 2014; DOI: https//doi.org/10.3399/bjgp14X680125.

39. Astin M, Griffin T, Neal RD, et al. The diagnostic value of symptoms for colorectal cancer in primary care: a systematic review. Br J Gen Pract 2011; DOI: https//doi.org/10.3399/bjgp11X572427.

40. Shapley M, Mansell G, Jordan JL, Jordan KP. Positive predictive values of $\geq 5 \%$ in primary care for cancer: systematic review. Br J Gen Pract 2010; DOI: https//doi.org/10.3399/bjgp10X515412.

41. Lewis G, Curry N, Bardsley M. Choosing a predictive risk model: a guide for commissioners in England. London: Nuffield Trust, 2011. https://www. nuffieldtrust.org.uk/files/2017-01/choosing-predictive-risk-model-guide-forcommissioners-web-final.pdf (accessed 19 Oct 2017).

42. Giles MF, Rothwell PM. Substantial underestimation of the need for outpatient services for TIA and minor stroke. Age Ageing 2007; 36(6): 676680.

43. Bos MJ, van Rijn ME, Witteman JM, et al. Incidence and prognosis of transient neurological attacks. JAMA 2007; 298(24): 2877-2885

44. Tuna MA, Li L, Tornada A, et al. The 12-year risk of stroke and coronary events after focal and non-focal transient neurological attacks: a populationbased study. Eur Stroke J 2016; 1(1 Suppl): 760

45. Tuna MA, Tornada A, Li L, et al. Short and long-term risk of stroke after a specialist diagnosis of TIA/minor stroke mimic: a population-based study. Int J Stroke 2015; 10(Suppl 2): 66.

46. Rothwell PM, Algra A, Chen Z, et al. Effects of aspirin on risk and severity of early recurrent stroke after transient ischaemic attack and ischaemic stroke: time-course analysis of randomised trials. Lancet 2016; 388(10042): 365-375. 